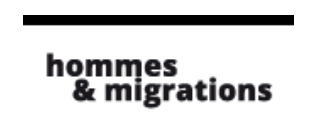

\section{Hommes \& migrations}

Revue française de référence sur les dynamiques

migratoires

1317-1318 | 2017

L'Europe en mouvement

\title{
Île du Monde
}

Un projet associatif pour connaître et valoriser les savoirs des migrants en Île-de-France

\section{Frida Calderon et Simone Tortoriello}

\section{(2) OpenEdition}

\section{Journals}

Édition électronique

URL : https://journals.openedition.org/hommesmigrations/3908

DOI : 10.4000/hommesmigrations.3908

ISSN : 2262-3353

Éditeur

Musée national de l'histoire de l'immigration

Édition imprimée

Date de publication : 1 avril 2017

Pagination : 140-143

ISBN : 978-2-919040-38-4

ISSN : $1142-852 X$

Référence électronique

Frida Calderon et Simone Tortoriello, « île du Monde », Hommes \& migrations [En ligne], 1317-1318| 2017, mis en ligne le 01 avril 2017, consulté le 28 juin 2022. URL : http://journals.openedition.org/ hommesmigrations/3908; DOI : https://doi.org/10.4000/hommesmigrations.3908 


\section{ÎLE DU MONDE : UN PROJET ASSOCIATIF POUR CONNAITTRE ET VALORISER LES SAVOIRS DES MIGRANTS EN ÎLE-DE-FRANCE}

Par FRIDA CALDERÓN (Anthropologue) et SIMONE TORTORIELLO (Sociologue) équipe de recherche de l'association

La présence de populations issues de l'immigration en Île-de-France est évidente pour nous tous. Mais quelles sont les habitudes et les pratiques quotidiennes de ces personnes venues d'ailleurs, au-delà du folklore et de l'exotisme ? Comment, grâce à nos différentes manières de voir le monde et de vivre au quotidien, pouvons-nous nous enrichir les uns les autres et partager nos différences ? L'association île du Monde a surgi de cette première curiosité : découvrir, au plus près de leur quotidien, les populations venues d'ailleurs et installées en île-de-France.

L 'Unesco définit le patrimoine culturel immatériel $(\mathrm{PCl})$ comme les pratiques, représentations, expressions, connaissances et savoir-faire - ainsi que les instruments, objets, artefacts et espaces culturels qui leur sont associés - que les communautés, les groupes et, le cas échéant, les individus reconnaissent comme faisant partie de leur patrimoine culturel. Ce patrimoine culturel immatériel, transmis de génération en génération, est recréé en permanence par les communautés et les groupes en fonction de leur milieu, de leur interaction avec la nature et de leur his- toire. II leur procure un sentiment d'identité et de continuité, contribuant ainsi à promouvoir le respect de la diversité culturelle et la créativité humaine. Le $\mathrm{PCl}$ se manifeste notamment dans les domaines suivants : les traditions et expressions orales, y compris la langue; les arts du spectacle ; les pratiques sociales, rituels et événements festifs ; les connaissances et pratiques concernant la nature et l'univers; les savoir-faire liés à l'artisanat traditionnel. Cette notion de patrimoine culturel immatériel nous a permis de délimiter ce qui allait devenir notre champ 
L'anthropologue et photographe d'île du Monde, Daniel Ortiz, lors de la célébration de la Holi à la Maison de l'Inde. @ DR.

d'observation, c'est-à-dire l'ensemble des pratiques et des gestes traditionnels accomplis par les différentes communautés migrantes. L'ajout du qualificatif translocal ${ }^{2}$ nous a permis de nous intéresser à leur caractère vivant et mouvant. L'objectif d'île du Monde s'est alors consolidé autour du projet de valorisation et de sauvegarde du patrimoine culturel immatériel translocal en île-de-France grâce à la présence de toutes les communautés migrantes.

Les membres de l'association ont commencé à photographier, à écouter et à enregistrer, parfois simplement à discuter avec les migrants franciliens : lors de grandes célébrations (par exemple, le Nouvel an Chinois ou le Lavage de la Madeleine), pendant la réalisation de pratiques traditionnelles (par exemple le temazcal mexicain ou la pachamanca péruvienne), en suivant des musiciens pour découvrir des musiques du monde (par exemple Miguel Ballumbrosio, maître du ca- jón péruvien, Pedro Kouyaté, griot mandingue, etc.) ou en approchant les sons des langues les plus variées. Un énorme registre audiovisuel s'est constitué dans cette première étape du travail. Mais comment faire connaître la diversité de ces pratiques venues d'ailleurs ? Est-ce que les Français savent que tous ces gestes et idées sont présents sur leur territoire? Un premier pas a consisté à promouvoir toute cette diversité. Pour cela, Île du Monde a créé un blog, un agenda événementiel en ligne, une page Facebook, une chaîne Youtube 3 , autant de moyens de communication accessibles permettant de développer une première voie de diffusion de la richesse que l'on commençait à découvrir. L'un des objectifs étant de faire connaître

moine culturel immatériel. Enjeux d'une nouvelle catégorie, Paris, Éditions de la Maison des sciences de l'homme, 2011. 3. Pour mieux nous connaître: www.iledumonde.org Afin de connaître notre production audiovisuelle : www.youtube.com/ user/iledumonde et pour nous suivre www.facebook.com/ileduMonde/4. Depuis ce premier projet en 2014, île du Monde collabore chaque année à la réalisation de l'inventaire du PCI. Nous avons réalisé à ce jour 7 fiches ethnographiques, accompagnées chacune d'une vidéo documentaire. Voir : http://www.culturecommunication.gouv.fr/Politiques-ministerielles/Patrimoine-culturel-immateriel/Inventaire-en-France/Inventaire/Fiches-de-I-inventaire-du-patrimoine-culturel-immateriel 


\section{INITIATIVE}

des activités culturelles d'origine étrangère se déroulant en île-de-France, ces outils nous ont d'abord permis d'atteindre un public large et varié. Mais, au-delà de la diffusion, nous cherchons à créer un objet visuel que le plus grand nombre puisse s'approprier. Internet offre dans ce sens la possibilité de visionner de manière simple et gratuite les documentaires réalisés par notre équipe, ce qui constitue pour nous le meilleur moyen de restitution aux communautés concernées.

Par ailleurs, certains membres de l'association étant sociologues et anthropologues, nous voulions réaliser un vrai travail de type ethnographique afin de mieux documenter tout cet univers mouvant et multiculturel. Nous avons élaboré et soumis un projet de recherche auprès du ministère de la Culture et de la communication afin de contribuer à la réalisation de l'inventaire du PCI français ${ }^{4}$. Nous avons fait le constat qu'il existait déjà des pratiques bien établies en France et que, par conséquent, certaines faisaient déjà partie

La démarche consiste alors

à aller à la rencontre des personnes, d'échanger avec

elles et de connaître leur point de vue sur les pratiques traditionnelles autant que sur l'intérêt de les faire exister en île-de-France. de l'inventaire du PCl national (par exemple le Jour des morts mexicain ou la Fête de Ganesh des Hindouistes), ce qui nous permettait de proposer l'élargissement de cet inventaire, en insistant justement sur le besoin de considérer les pratiques venues d'ailleurs. La Fête du Printemps asiatique, la danse du lion, le Lavage de la Madeleine, les chants polyphoniques géorgiens, l'art des griots mandingues sont autant de pratiques réalisées et transmises en Île-de-France par les migrants des différentes communautés depuis plusieurs générations : il est par conséquent légitime de les intégrer dans cet inventaire.
Afin de valoriser ces différentes pratiques, île du Monde a développé une méthodologie de travail où l'on donne la priorité à la participation des communautés elles-mêmes concernées dans la définition de leur patrimoine culturel. La démarche consiste alors à aller à la rencontre des personnes, d'échanger avec elles et de connaître leur point de vue sur les pratiques traditionnelles autant que sur l'intérêt de les faire exister en Île-de-France. La captation vidéo fait partie des outils privilégiés par notre association. Grâce à la réalisation de vidéos documentaires, nous élaborons un témoignage visuel accessible à tous servant aussi d'outil de restitution auprès des communautés.

La rencontre avec les communautés migrantes nous a permis de constater les difficultés liées à ces populations en termes d'expression et de valorisation de leurs savoirs et savoir-faire traditionnels. Considérant ce contexte, le projet "Rencontres île du Monde » vise à stimuler des rencontres interculturelles et la création de lien social. Le projet consiste à organiser des repas réalisés par des personnes d'origine étrangère chez elles auxquels est convié le public intéressé par la découverte culturelle : un prétexte pour créer autour de la cuisine un cadre convivial qui permette l'échange et la discussion entre des personnes de différentes cultures. Aujourd'hui, une vingtaine de cuisiniers amateurs d'origines diverses, habitant dans les quartiers prioritaires du XVIII ${ }^{e}$ arrondissement $^{5}$, participent à ce projet. Le site Internet de l'association est devenu, dans ce cadre, une vitrine pour les cuisiniers, grâce à des vidéos de présentation de chacun d'entre eux et de leurs recettes traditionnelles, ainsi qu'un espace de mise en relation avec des clients potentiels. 
Le $\mathrm{PCl}$ est un concept qui permet de travailler sur la sauvegarde de pratiques culturelles, en favorisant la valorisation effective de ces pratiques. Dans ce sens, île du Monde a construit un dispositif d'accueil, d'intégration et d'insertion professionnelle des porteurs de tradition. Le projet « Rencontre île du Monde " nous a donnés de l'inspiration : parmi les cuisiniers amateurs que nous avons rencontrés, nous nous sommes aperçus que certains avaient un savoir-faire important et surtout l'envie de le transformer en une profession. Le projet " Île des savoirs", le dernier mis en place par l'association, a pour but de répondre à cette nécessité. L'association met en place un parcours d'accompagnement vers l'insertion professionnelle en considérant les projets personnels de chacun en relation avec leur savoir culinaire. À ce jour, cing cuisiniers de différentes origines font partie de ce projet et participent aux activités d'une entreprise d'insertion qui prend la forme d'un traiteur solidaire et multiculturel.

L'objectif de l'association est aujourd'hui de réaliser un vrai diagnostic des savoir-faire présents dans les communautés issues des diasporas en île-de-France et d'utiliser le même modèle, mis en place pour la gastronomie, pour l'insertion dans les métiers liés à d'autres types de savoir, comme l'artisanat, le bien-être traditionnel, les sports, les langues minoritaires, etc. La création d'un centre de ressources dans lequel pourront se retrouver tous ces différents savoirs et toutes les différentes activités de l'association constitue l'étape suivante dans le déroulement de notre travail.

L'association jouit aujourd'hui d'une équipe de travail multidisciplinaire : Frida Calderon Bony, Simone Tortoriello et Daniel Ortiz Avila sont les responsables des activités de recherche ethnographique et de la réalisation d'articles et de documentaires vidéo ; Carlo Barletta s'occupe du déve-

loppement du projet d'accompagnement vers l'insertion professionnelle et de l'économie sociale et solidaire ; Pepe Pastor, l'initiateur de l'association, accomplit le rôle de médiateur culturel et Stéphanie Magalage soigne tous les aspects liés à la communication. Par ailleurs, des bénévoles et des stagiaires contribuent de manière ponctuelle à la réalisation de différentes activités.

île du Monde profite également du soutien de différents partenaires pour la réalisation de ses activités, comme le ministère de la Culture et de la communication, la Mairie de Paris, la Fondation de France. L'association a développé un réseau de partenaires très hétéroclites qui vont de dizaines d'associations d'origine étrangère à des établisse-

La création d'un centre de ressources dans lequel pourront se retrouver tous ces différents savoirs et toutes les différentes activités de l'association constitue l'étape suivante dans le déroulement de notre travail. ments universitaires tels que l'Inalco ou l'université Paris $V$, en passant également par de nombreuses structures de proximité, tels que des centres sociaux, des espaces dédiés à l'emploi ou à la formation.

Dans son ensemble, le travail accompli par île du Monde constitue un nouveau dispositif applicable à l'accueil et à l'intégration des migrants. Un dispositif qui favorise un regard positif sur la migration considérée comme une richesse à connaître et à apprécier. À travers ses différentes activités, l'association a pour but de permettre aux migrants de s'intégrer professionnellement et socialement en capitalisant leurs savoir-faire traditionnels.

La valorisation de la diversité culturelle est toujours nécessaire et île du Monde répond à ce besoin grâce à la recherche ethnographique, la promotion de rencontres interculturelles et la valorisation concrète des savoir-faire issus des diasporas. 